\title{
Aproximación comparativa inicial en resultados del WISC-III v.ch. entre una muestra de jóvenes escolarizados pertenecientes a zonas rurales de la XV Región de Arica y Parinacota y la norma nacional*
}

\author{
Initial comparative study of the results obtained in a Chilean version of WISC-III \\ between a sample of young students belonging to rural areas of the XV Region \\ of Arica y Parinacota and the national standard
}

\author{
Constanza Veloso, ${ }^{a}$ Alejandro Cuadra, ${ }^{a b}$ Roberto Storey, ${ }^{a c}$ \\ Roberto González, ${ }^{d}$ Benjamín Moraga ${ }^{e}$ \\ a Universidad de Tarapacá \\ Telf.: 987191137. Correo electrónico: veloso.constanza@gmail.com \\ b Telf.: 998662841. Correo electrónico: acuadra@uta.cl \\ c Telf.: (56) 582205820. Correo electrónico: rstorey@uta.cl \\ ${ }^{\mathrm{d}}$ Telf.: 977977482. Correo electrónico: rgonzalezvilchez@ gmail.com \\ eTelf.: 992100885. Correo electrónico: begnobilis@gmail.com
}

\begin{abstract}
El objetivo general de esta investigación fue comparar los resultados de la adaptación chilena del WISC-III obtenidos en una muestra de 94 jóvenes pertenecientes a zonas rurales de la XV Región de Arica y Parinacota con la norma nacional. Además, se decidió incluir una medición adicional de coeficiente intelectual, el test de inteligencia no verbal TONI-2, ya que no se encuentra afecto a sesgo lingüístico, motriz o cultural. Los hallazgos de esta investigación evidencian que el coeficiente intelectual de los jóvenes muestreados fue significativamente menor en todas las escalas e índices de la versión chilena del WISC-III respecto de la norma nacional. Sin embargo, no se hallaron diferencias estadísticamente significativas en el coeficiente intelectual cuando fue medido a través del TONI-2. Se discuten las implicancias prácticas de estos resultados.
\end{abstract}

RESUMEN

Palabras clave: WISC-III versión chilena, ruralidad, inteligencia, coeficiente intelectual, jóvenes.

\section{ABSTRACT}

The overall objective of this research was to compare the results of the Chilean adaptation of WISC-III obtained in a sample of 94 young people, from rural areas of XV Region of Arica y Parinacota, with the national standard. Furthermore, it was decided to include an additional measurement of IQ, the nonverbal intelligence test TONI-2, since it is not subject to linguistic bias, motor or cultural. The findings of this research showed that the IQ of the sampled young people was significantly lower on all scales and indexes of the Chilean version of WISC-III in relation to the national standard. However, no statistically significant differences were found in IQ, when it was measured by TONI-2. The practical implications of these results are discussed.

Key words: WISC-III Chilean version, rurality, intelligence, IQ, youth.

Se agradece el apoyo al Convenio de Desempeño MINEDUC-UTA. 
Estudios Pedagógicos XLII, N 3: 413-427, 2016

APROXIMACIÓN COMPARATIVA INICIAL EN RESULTADOS DEL WISC-III V.CH. ENTRE UNA MUESTRA DE JÓVENES ESCOLARIZADOS PERTENECIENTES A ZONAS RURALES DE LA XV REGIÓN DE ARICA Y PARINACOTA...

\section{INTRODUCCIÓN}

La sociedad humana valora altamente la inteligencia considerándola un factor decisivo para los logros educativos, el éxito en el trabajo y para el desarrollo socioeconómico de los pueblos (Ardila, 2011). Un aspecto de la inteligencia es la capacidad intelectual (Ramírez y Rosas, 2007). Respecto de la forma de medir la capacidad intelectual, las escalas Wechsler, a lo largo del tiempo, se han trasformado en una de las formas más utilizadas para cuantificarla en el mundo occidental. Esta supervivencia de más de seis décadas, tanto de la versión para niños como para la de los adultos, ha sido facilitada por la reiterada actualización del material de toma y de las normas poblacionales, así como por el elevado número de investigaciones que han validado su utilidad en las distintas áreas del quehacer psicológico (Frank de Verthelyi, 2000).

Específicamente, entre 1975 y 1980, la escala de inteligencia para niños de Wechsler (WISC-R) fue adaptada y estandarizada para el Área Metropolitana de Santiago de Chile por tres grupos de investigación (Adriazola et al., 1976; Calderón et al., 1980; Cañas et al., 1978). Sin embargo, el desarrollo de los medios de comunicación de masas, las modificaciones curriculares del sistema educacional y el mayor acceso a la educación han dejado obsoleto el WISC-R, puesto que los avances señalados han permitido que los niños tengan en la actualidad una mayor visión y conocimiento del mundo (Ramírez y Rosas, 2007). En consecuencia, surgió la necesidad de una nueva versión del test (WISC-III), con el fin de contar con un instrumento válido y confiable que permitiera el diagnóstico adecuado de los niños chilenos del nuevo milenio (Ramírez y Rosas, 2007). Si bien esta escala mantiene las características fundamentales del WISC-R, proporciona datos normativos, materiales, contenidos y procedimientos de administración actualizados (Wechsler, 1991, 1997).

Es importante señalar que en la estandarización del WISC-III v.ch. se afirmó que tanto la magnitud de la muestra como el hecho de que se basó en un muestreo estratificado (por regiones del país) permite confiar en que las normas son representativas de las diferentes especificidades sub-culturales que puedan existir en el país (Ramírez y Rosas, 2007), aunque el espacio muestral de las regiones utilizadas - metropolitana (785), IV región (352), V región (557) y la IX región (230) en un total de 1914 niños- no incluyó ninguna localidad rural.

Además de los datos normativos y avances señalados por los autores de la estandarización del WISC-III en Chile, es necesario dar respuesta a la creciente demanda de evaluación multi-subcultural, teniendo en cuenta que toda técnica de evaluación es el resultado de una cultura y responde a los valores de esta última (Cayssials, 1998). En esta línea, los test de inteligencia en general toman como referencia a la población más o menos escolarizada de la civilización occidental y predominantemente urbana (Cayssials, 1998). Por ello se propone que la versión estandarizada del WISC-III en Chile, por su implicancia educacional, debe aplicarse a niños de escuelas rurales con el objetivo de comparar sus resultados con el estándar nacional, máxime si se considera que las habilidades que evalúa el WISC-III son valoradas de diversa manera en distintas culturas.

De acuerdo a todo lo expuesto anteriormente, el objetivo general de esta investigación fue comparar los resultados de la aplicación del WISC-III v.ch. en una muestra de jóvenes escolarizados pertenecientes a zonas rurales de la XV Región, con la norma nacional. Además, se decidió incluir una medición adicional de coeficiente intelectual, el test de 
inteligencia no verbal TONI-2, dado que no se encuentra afecto a sesgo cultural. En consecuencia, si el WISC-III v.ch es sensible a sesgo cultural los resultados entre la muestra rural y norma nacional debieran diferir significativamente, mientras que los puntajes del TONI-2 entre la muestra rural y la norma no deberían presentar una diferencia estadísticamente significativa.

La utilidad de esta investigación radica en que, de no encontrarse diferencias entre la muestra rural y la norma nacional del WISC-III v.ch., podría continuar utilizándose dicha norma sin riesgo de generar interpretaciones sesgadas del coeficiente intelectual en niños y niñas del área rural. Por el contrario, si el estudio arroja diferencias significativas debiera reconsiderarse la utilización de la norma nacional a la hora de evaluar a esta subpoblación e interpretar, por ahora, con precaución los resultados que arroja el WISC-III v.ch.

\section{INTELIGENCIA, MEDICIÓN Y ESCALAS WECHSLER}

A lo largo del tiempo se han desarrollado numerosas teorías sobre la inteligencia (Teoría de Dos Factores de Spearman; Teoría Multi-Factor de Thorndike; Teoría de Factor Grupal; Teoría de Estructura Tridimensional de Guilford; Teoría Jerárquica de Stenberg; Teoría de Piaget; Teoría de Inteligencias Múltiples de Gardner, etc.), de los aspectos que la forman y afectan, y estos han sido examinados desde varios ángulos (Toker, 1968). Aunque la gran mayoría de los científicos está de acuerdo en que la inteligencia es medible, no han podido ofrecer una definición única sobre esta misma (Walsh y Betz, 1995).

El primer intento de medir la inteligencia a través de un test lo realizó Alfred Binet en 1905, quien diseñó una serie de situaciones problema que poseían dificultad gradual creciente y clasificó las que podían ser resueltas por niños de diversas edades. En esta forma se propuso una secuencia que dio origen al concepto de "edad mental", diferente de la "edad cronológica". Wilhelm Stern dividió la edad mental entre la edad cronológica para obtener un "cociente mental"; Edad mental/Edad cronológica. Más tarde, Lewis Terman multiplicó el cociente mental por cien para eliminar la fracción y es así como emergió el Coeficiente Intelectual (CI); Edad mental/Edad cronológica x 100. Más adelante, se propusieron otras pruebas para medir CI, entre las cuales se encontraban las escalas de Wechsler en sus diversas formas (WAIS, WISC, WPPSI).

Según Wechsler, la inteligencia es un constructo hipotético y se refiere a la capacidad agregada o global de la persona para actuar con un propósito, pensar racionalmente e interactuar en forma efectiva con su medio ambiente (Matarazzo, 1972). Wechsler señaló que su escala de inteligencia no abarcaba toda la inteligencia y que algunas pruebas apelaban a fenómenos no intelectuales, tales como la planificación y la conciencia de los objetivos, el entusiasmo, la dependencia y la independencia en cierto campo, la impulsividad, la ansiedad y la persistencia (Wechsler, 1991, 1997). En consideración a que la capacidad intelectual es únicamente un aspecto de la inteligencia, Wechsler exhorta a las personas que interpretan los resultados de un test de inteligencia a distinguir entre el CI que arroja el test, por un lado, y la inteligencia, por otro (Wechsler, 1979 Cit. en Wechsler, 1991, 1997).

Una de las primeras escalas fue publicada en 1939, cuando Wechsler era psicólogo del Hospital Bellevue de Nueva York, por lo que es conocida con el nombre de Wechsler Bellevue. Su primera utilización fue en procesos de selección de personal y tenía dos versiones: las formas I y II. En 1949 Wechsler adaptó la forma II, que dio como resultado 
Estudios Pedagógicos XLII, N 3: 413-427, 2016

APROXIMACIÓN COMPARATIVA INICIAL EN RESULTADOS DEL WISC-III V.CH. ENTRE UNA MUESTRA DE JÓVENES ESCOLARIZADOS PERTENECIENTES A ZONAS RURALES DE LA XV REGIÓN DE ARICA Y PARINACOTA...

la primera Escala de Inteligencia de Wechsler para Niños (Wechsler Intelligence Scale for Children, WISC), con un rango de aplicación entre los 5 y 15 años. Esta escala consistía en 12 sub-pruebas, agrupadas en dos secciones, verbal y manual, aplicadas en el siguiente orden: Información general, comprensión general, aritmética, similitudes, vocabulario, dígitos, completación de imágenes, ordenamiento de historias, bloques, ensamblaje de objetos, códigos y laberintos; dígitos y laberintos son pruebas auxiliares.

Posteriormente, es actualizada y revisada en 1974, dando origen al WISC-R, los principales cambios fueron la expansión del rango de edad de 6 a 16 años, la aplicación y puntuación de las sub-pruebas (Cronbach, 1990; Savasir y Sahin, 1995) y la completación de imágenes que fue alterada completamente. Esta versión se mantuvo vigente durante diecisiete años, hasta 1991, dando lugar a una nueva versión WISC-III, la cual mantiene las características fundamentales de su versión anterior, pero que, a su vez, proporciona normas, materiales, contenidos y procedimientos de administración actualizados. El WISC-R y WISC-III coinciden hasta un 72\% aproximadamente; con esta revisión (WISCIII) los elementos se han vuelto más coloridos, más sensibles al género y la discriminación racial (Kezer y Arik, 2012).

El Test de Inteligencia para niños de Wechsler Tercera Edición (WISC-III) es un instrumento clínico de administración individual para evaluar la capacidad intelectual de niños de 6 años a 16 años y 11 meses de edad. El WISC-III consta de varias sub-pruebas, cada una de las cuales mide una faceta diferente de la inteligencia. Al igual que el WISC-R, el desempeño del niño en las diferentes sub-pruebas se resume en tres puntajes compuestos, a saber: el coeficiente intelectual Verbal, de Ejecución y Total, que proveen estimaciones de la capacidad intelectual del individuo.

Esta escala comprende las 12 sub-pruebas del WISC-R organizadas en dos subgrupos, las de CI Verbal: Información, Analogías, Aritmética, Vocabulario, Comprensión y Retención de Dígitos; y las de CI de Ejecución: Completación de Figuras, Claves, Ordenamiento de Historia, Construcción con Cubos, Ensamblaje de Objetos, Laberintos; se agrega una subprueba nueva: Búsqueda de Símbolos.

Además de los puntajes de CI Verbal, CI de Ejecución y CI Total, pueden calcularse cuatro puntajes índices basados en factores: 1) Comprensión Verbal, 2) Organización Perceptual, 3) Velocidad de Procesamiento y 4) Ausencia de Distractibilidad.

\section{PERSPECTIVA TRANSCULTURAL Y DISTANCIA CULTURAL}

La psicología ha empleado tradicionalmente el término "medio ambiente" en lugar de la cultura. El medio ambiente, como la cultura, es también un concepto teórico muy amplio, lo que potencialmente puede incluir todas las características del entorno físico y social. La psicología ha empleado generalmente definiciones operativas de los elementos estructurales sociales, como el ajuste rural-urbano, nivel socioeconómico, nivel educativo, etc. Se ha encontrado que algunas de estas variables ambientales están consistentemente relacionadas con la inteligencia y el funcionamiento cognitivo. Por ejemplo, el nivel socioeconómico se correlaciona altamente con el desempeño en las pruebas de inteligencia. Otro correlato de la inteligencia es el número de años de escolaridad de la persona; las personas con más años de escolaridad obtienen mejores resultados en las pruebas de inteligencia que aquellos con menos años. Otro hallazgo típico es el nivel de la educación de los padres, el 

JÓVENES ESCOLARIZADOS PERTENECIENTES A ZONAS RURALES DE LA XV REGIÓN DE ARICA Y PARINACOTA...

cual correlaciona con el rendimiento de los niños en las pruebas de inteligencia. También se han establecido relaciones con los estilos de crianza de los hijos, el clima de la familia, maestros y niños, etc. (Georgas et al., 2003).

Las culturas definen la inteligencia de manera diferente, de acuerdo a sus propias demandas ecológicas y sociales. También hay variaciones en los tipos de procesos cognitivos que son importantes para la adaptación a las exigencias ecológicas de cada cultura (Georgas et al., 2003). Según Sternberg (1992) el rol de la inteligencia en el manejo de lo novedoso, así como en la automatización del procesamiento de la información, es una característica universal, pero las manifestaciones de estos componentes en la experiencia deben de considerarse relativos a cada contexto. Aquello que constituye un pensamiento o conducta adaptativa en una cultura no lo es necesariamente en otra. Más aún, pensamientos y acciones que pueden ser apropiados para la modelización de la conducta en un contexto pueden no serlo para otro. En esta misma línea, Miller (1997) señala que las diferentes culturas y subculturas no solo definen la inteligencia acorde a qué es considerado adaptativo para su particular nicho ecológico; también promueven el desarrollo de competencias disímiles, y, por lo tanto, las conductas consideradas inteligentes son culturalmente relativas.

En relación con lo anterior, las habilidades que evalúa el WISC-III son valoradas de diversa manera en distintas culturas. Los test de inteligencia, en general, toman como referencia a la población más o menos escolarizada de la civilización occidental, predominantemente urbana, verbal, técnica, industrial y competitiva (Cayssials, 1998). Como la mayoría de las baterías de exploración de la inteligencia, el WISC-III está sometido a la influencia de fuertes factores culturales. La propia estructura de la técnica, en tanto incluye la resolución de tareas verbales, ya lo ha hecho blanco de fuertes críticas por el sesgo cultural que supone. Sin embargo, este no es el único aspecto donde la diversidad cultural no es contemplada, también lo es el análisis cuantitativo elemental, el cual adolece de igual carencia (Martínez, 2009).

Una forma de abordar las diferencias culturales puede ser desde el enfoque de la Distancia Cultural, el cual se describe como una amalgama entre los puntos de vista de la psicología ambiental y social. Sostiene que cualquier subcultura que funciona de acuerdo a principios que no están presentes o igualmente operativos en la cultura mayoritaria, procesa la información funcional diferente a la cultura mayoritaria. Por lo tanto, el rendimiento de la minoría en pruebas basadas y validadas para la cultura mayoritaria mostrará patrones de respuesta diferentes a la norma (Grubb, 1983).

Otro enfoque relacionado con el anterior, que puede abordar las diferencias culturales, es la Psicología Transcultural, que estudia la relación entre la cultura y las variables psicológicas, es decir, se ocupa de dos cuestiones acerca de esta relación: la primera cuestión es determinar el grado de comunalidad que existe en los procesos psicológicos, o universales, a través de las culturas; la segunda cuestión consiste en el grado de variabilidad de los procesos psicológicos debido a influencias culturales específicas. Por lo tanto, uno de los objetivos de la psicología transcultural es entender la relación entre el comportamiento humano y los contextos culturales en los cuales se expresa y desarrolla (Berry et al., 2002). La mayoría de los estudios transculturales describe las similitudes y diferencias entre culturas en los fenómenos psicológicos (van Hemert, 2003). La psicología transcultural propone que existe una creciente conciencia de la existencia de valores culturales heterogéneos, no solamente en distintos continentes y entre países que conforman un mismo continente, sino también entre regiones que integran un mismo país (Cayssials, 1998). 
Estudios Pedagógicos XLII, N 3: 413-427, 2016

APROXIMACIÓN COMPARATIVA INICIAL EN RESULTADOS DEL WISC-III V.CH. ENTRE UNA MUESTRA DE JÓVENES ESCOLARIZADOS PERTENECIENTES A ZONAS RURALES DE LA XV REGIÓN DE ARICA Y PARINACOTA...

Aunque hay muchas diferencias de opinión en cuanto a qué es la inteligencia, la mayoría de los psicólogos transculturales estarían de acuerdo en que las manifestaciones de los procesos cognitivos están incrustadas en la cultura. Es decir, a pesar de que los procesos cognitivos son universales en todas las culturas, las diferentes manifestaciones de estos procesos cognitivos representan diferentes tipos de adaptaciones cognitivas a las exigencias ecológicas específicas y a los patrones ecoculturales (Berry et al., 2002).

Más allá de los recaudos necesarios para una adecuada evaluación transcultural, el creciente fenómeno de globalización y la incrementada demanda de instrumentos "transculturables" para medir inteligencia han dado lugar a dos respuestas a la vez disímiles y complementarias: 1) Énfasis en la traducción y adaptación de las técnicas existentes a múltiples idiomas y culturas; 2) Aparición de nuevas técnicas no verbales consideradas más libres de sesgo cultural (Frank de Verthelyi, 2000).

\section{EL TEST DE INTELIGENCIA NO VERBAL TONI}

La diversidad cultural y de lenguaje pone de relieve la necesidad de mediciones no verbales de la capacidad cognitiva (Roid y Miller, 1997). Existe una clara necesidad de pruebas de inteligencia que reduzcan la influencia del lenguaje y la fluidez del idioma para una puntuación más precisa, debido a que las mediciones no verbales de la capacidad cognitiva producen resultados semejantes para dos personas con inteligencia similares, incluso si uno domina el idioma y el otro no (Hopper, 2000).

Las pruebas no verbales de inteligencia se han desarrollado y utilizado para satisfacer las necesidades de evaluación educacional de individuos con dominio limitado del idioma dominante y que no pueden ser adecuadamente evaluados con pruebas de inteligencia tradicionales basadas en el lenguaje (Bracken y McCallum, 1997).

El objetivo en el diseño de las pruebas no verbales es reducir el sesgo en la influencia del idioma y las diferencias culturales en la puntuación, aumentando así la imparcialidad de la prueba. Por consiguiente, un test de inteligencia debería evaluar la inteligencia, no la ansiedad, la habilidad del lenguaje o el grado de familiarización con la cultura dominante. Entonces, cuando la prueba no evalúa en ciertas poblaciones lo que debería evaluar, se pueden configurar constructos irrelevantes. Del mismo modo, los exámenes que requieren instrucciones verbales, el conocimiento y la resolución de un problema verbal y una respuesta verbal, crean preocupación por la irrelevancia de constructo en la evaluación de personas que son diferentes a la cultura mayoritaria (Hopper, 2000). Ahora bien, las pruebas no verbales de inteligencia pueden ser una alternativa viable, a pesar de las limitaciones inherentes que conllevan (Lopez, Lamar y Scully-Demartini, 1997).

El test de inteligencia no verbal es claramente una prueba de inteligencia no verbal, libre de lenguaje, de hecho existen numerosas razones para llamarlo test de inteligencia no verbal, comenzando por su contenido, el formato de instrucciones y el formato de respuestas, los cuales no requieren leer, escribir, hablar ni escuchar (Hopper, 2000). En esta línea, los test de inteligencia no verbal reducen el uso del lenguaje en el proceso de administración (Anastasi y Urbina, 1997), empleando frecuentemente mímica o gestos parar dar las instrucciones y permitiendo dar respuestas gestuales (Hopper, 2000). Estas características hacen que las pruebas no verbales sirvan a profesionales que luchan con la dificultad de evaluar a individuos con bajo dominio del idioma o diferencias culturales. 
En consecuencia, algunas poblaciones como los discapacitados auditivos, personas que no hablan el idioma y las culturas de las minorías étnicas se beneficiarían de las pruebas que no se basan en los significados verbales de la cultura mayoritaria (Kowall, Watson y Madak, 1990).

Jensen (1980) indica algunas normas y dimensiones que deben cumplir los test libres de influencias culturales o de lenguaje: a) Deben ser medidas de tipo manipulativo que puedan sustituir a las de papel y lápiz; b) Las instrucciones se transmitirán por medio de mímica y no de forma oral o escrita; c) Deben incluir algunos ejemplos previos de práctica; d) No deben tener tiempo limitado; e) En lugar de estar formados por dibujos concretos o fragmentos de lectura, el contenido de los elementos debe ser de tipo abstracto; f) Para contestar al test se requerirá razonamiento o capacidad para resolver problemas, sin que sea necesario tener que recordar ninguna información específica; g) Los problemas deben ser nuevos, para evitar contaminación por el recuerdo de una información aprendida previamente.

Al construir el TONI primera versión se procuró que cumpliera todas estas normas. A pesar de la amplia utilización de los test de inteligencia y de que se puede disponer de muchos instrumentos cuya validez ha sido comprobada teórica y empíricamente, se observa una necesidad creciente de instrumentos de este tipo que no estén excesivamente afectados por factores lingüísticos, motrices o culturales (Brown, Sherbenou y Johnsen, 1995).

El TONI-2 mide la capacidad para resolver problemas con figuras abstractas, exento de la influencia del lenguaje. Se diseñó para ser utilizado con sujetos de edades comprendidas entre 5 y 85 años de edad. Existen dos formas equivalentes: A y B; cada una de ellas está formada por 55 elementos ordenados por dificultad creciente (Brown et al., 1995).

Cabe hacer notar que se han construido muy pocos test que no tengan carga cultural. Ciertamente, ningún test puede estar completamente libre de la influencia de la cultura, pues la propia aplicación de pruebas lleva consigo una importante carga cultural (Brown et al., 1995). Sin embargo, el número de personas de diversas culturas, tanto en los centros de trabajo como en la escuela, va en aumento y esto hace que sea necesario disponer de medidas para apreciar la capacidad intelectual que dependan lo menos posible de la influencia de aspectos específicos del lenguaje, minimizado de este modo el sesgo respecto a sujetos de minorías étnicas o distinto nivel socio-económico o lingüístico (Sattler, 1992).

\section{MÉTODO}

\subsection{OBJETIVO GENERAL}

Comparar los resultados de la aplicación del WISC-III v.ch. en una muestra de jóvenes escolarizados pertenecientes a zonas rurales de la XV Región, con la norma nacional.

\subsection{OBJETIVOS ESPECÍFICOS}

- Comparar los resultados del WISC-III v.ch. de niños que pertenecen a escuelas de localidades rurales con la norma nacional.

- Comparar los resultados del TONI-2 de niños que pertenecen a escuelas de localidades rurales con la normativa establecida por dicho test. 


\subsection{PARTICIPANTES}

Los participantes fueron 94 jóvenes, hombres y mujeres, con un rango de edad que osciló entre los 6 y 16 años, todos estudiantes de escuelas municipales ubicadas en zonas rurales de la XV región de Arica y Parinacota. Las localidades comprendidas para este estudio fueron: Visviri, Cosapilla y Guacoyo, de la comuna de General Lagos; Codpa, Guañacagua y Chitita, de la comuna de Camarones; y Sobraya, de la comuna de Arica. La técnica de muestreo empleada fue de tipo no probabilística, por disponibilidad.

\subsection{VARIABLES}

- $\quad$ Factor: Ruralidad frente a norma poblacional.

- Variable dependiente: Resultados de los instrumentos WISC-III v.ch. y TONI-2.

\subsection{INSTRUMENTOS}

Escala Wechsler de Inteligencia para Niños, Tercera Edición, Versión Chilena (WISCIII v.ch.). Tiene como objetivo la evaluación de la inteligencia en niños que se encuentran en etapa escolar, desde los 6 años y 0 meses hasta los 16 años y 11 meses.

Esta batería de evaluación psicológica arroja como resultados un CI Total, CI Verbal y CI Ejecutivo del niño evaluado, además de cuatro índices factoriales conformados por 13 subpruebas, a saber: comprensión verbal, organización perceptual, velocidad de procesamiento y ausencia de distractibilidad. A partir de sus resultados cuantitativos es posible hacer análisis de armonía de rendimiento y comparaciones entre e intra-sujeto.

Su aplicación es de utilidad en el contexto clínico, neuropsicológico, educacional y jurídico-forense con el fin de tipificar el coeficiente intelectual de niños y adolescentes, así como también comprende el perfil de funciones cognitivas. Al aplicar WISC-III v.ch. se podrá tipificar el nivel cognitivo del evaluado con dos niveles de análisis: la comparación con sujetos de su misma edad y nivel educativo y la comparación consigo mismo. Prueba de 90 minutos para aplicación individual.

Tabla 1. Interpretación cualitativa de los puntajes de CI WISC-III v.ch.

\begin{tabular}{|l|l|}
\hline \multicolumn{1}{|c|}{ CI } & \multicolumn{1}{c|}{ Clasificación } \\
\hline 130 y más & Muy superior \\
\hline $120-129$ & Superior \\
\hline $110-119$ & Media alta \\
\hline $90-109$ & Promedio \\
\hline $80-89$ & Media baja \\
\hline $70-79$ & Limítrofe \\
\hline 69 y menos & Intelectualmente deficiente \\
\hline
\end{tabular}



JÓVENES ESCOLARIZADOS PERTENECIENTES A ZONAS RURALES DE LA XV REGIÓN DE ARICA Y PARINACOTA...

La consistencia interna de las sub escalas oscila entre .65 en Dígitos a .91 en Vocabulario. En relación al CI de ejecución, la consistencia interna es de .75, y para CI Verbal fue de .85; la consistencia interna de la escala total es de .87 (Ramírez y Rosas, 2007).

Test de Inteligencia No Verbal Apreciación de la Habilidad Cognitiva Sin Influencia del Lenguaje (TONI-2): El TONI-2 es una medida de la capacidad para resolver problemas con figuras abstractas, exenta de la influencia del lenguaje. Se diseñó para ser utilizado con sujetos de edades comprendidas entre 5 y 85 años de edad. Existen dos formas equivalentes: A y B; cada una de ellas está formada por 55 elementos ordenados por dificultad creciente.

Tabla 2. Términos de diagnóstico para interpretar los puntajes del TONI-2

\begin{tabular}{|l|l|}
\hline \multicolumn{1}{|c|}{ CI } & \multicolumn{1}{c|}{ Clasificación } \\
\hline 130 y más & Muy superior \\
\hline $121-130$ & Superior \\
\hline $111-120$ & Medio alto \\
\hline $90-110$ & Medio \\
\hline $80-89$ & Medio bajo \\
\hline $70-79$ & Bajo \\
\hline 70 y menos & Muy bajo \\
\hline
\end{tabular}

\subsection{PROCEDIMIENTO}

En primera instancia, concurrimos a la asociación de municipalidades rurales de General Lagos y Camarones para establecer contacto con las autoridades pertinentes, con el fin de explicar los objetivos del estudio, realizar un catastro de los establecimientos educacionales disponibles y solicitar autorización para acceder a la muestra. Se realizó este mismo procedimiento con la escuela España ubicada en la localidad de Sobraya, que se encuentra dentro de la comuna de Arica.

Una vez obtenida la autorización, el apoyo de los alcaldes, el de los Departamentos de Administración de Educación Municipal y los profesores a cargo de cada establecimiento, se dividió el proceso de aplicación de las baterías de evaluación en tres etapas. En primer lugar, emprendimos viaje desde Arica a las escuelas de la comuna de General Lagos (Internado Visviri, Escuela Cosapilla y Escuela Guacoyo), ubicada en valores aproximados a $287 \mathrm{~km}, 6$ horas y a una altura de 4000 m.s.n.m. En segundo lugar, acudimos a las escuelas de la comuna de Camarones (Liceo Valle de Codpa, Escuela Guañacagua y Escuela Chitita) ubicada aproximadamente a $120 \mathrm{~km}$ y 2 horas con 30 minutos; y por último a la Escuela G-28 España, la cual se encuentra a $30 \mathrm{~km}$ y 40 minutos de la ciudad.

La aplicación de los instrumentos fue de forma individual, en salas que cumplían los requisitos necesarios en cuanto a espacio, silencio y comodidad, las cuales fueron facilitadas por los distintos establecimientos. 
Estudios Pedagógicos XLII, N 3: 413-427, 2016

APROXIMACIÓN COMPARATIVA INICIAL EN RESULTADOS DEL WISC-III V.CH. ENTRE UNA MUESTRA DE JÓVENES ESCOLARIZADOS PERTENECIENTES A ZONAS RURALES DE LA XV REGIÓN DE ARICA Y PARINACOTA...

La participación de los estudiantes fue voluntaria con la seguridad de que se protegería la identidad, manteniendo el anonimato de quienes colaboraron. Cabe señalar que la batería de instrumentos constó de WISC-III v.ch. y TONI-2.

\section{RESULTADOS}

En la Tabla 3 se presenta la distribución de la muestra en porcentajes según los criterios de clasificación de cada instrumento.

Tabla 3. Clasificación de la muestra según puntajes WISC-III v.ch. y TONI-2

\begin{tabular}{|c|c|c|c|c|c|c|c|}
\hline \multicolumn{3}{|c}{ WISC-III v.ch. } & \multicolumn{4}{c|}{ TONI-2 } \\
\hline$\Sigma$ & Clasificación & $\%$ & $N$ & $N$ & $\%$ & Clasificación & $\Sigma$ \\
\hline \multirow{4}{*}{$32 \%$} & Muy superior & $2.12 \%$ & 2 & 2 & $2.12 \%$ & Muy Superior & \\
\cline { 2 - 8 } & Superior & $1.06 \%$ & 1 & 3 & $3.19 \%$ & Superior & \\
\cline { 2 - 8 } & Media alta & $6.38 \%$ & 6 & 18 & $19.14 \%$ & Media alta & \multirow{2}{*}{$67 \%$} \\
\cline { 2 - 8 } & Promedio & $22.34 \%$ & 21 & 40 & $42.55 \%$ & Media & \\
\hline \multirow{3}{*}{$68 \%$} & Media Baja & $15.95 \%$ & 15 & 21 & $22.34 \%$ & Media baja & \\
\cline { 2 - 7 } & Limítrofe & $31.91 \%$ & 30 & 7 & $7.44 \%$ & Baja & \multirow{2}{*}{$33 \%$} \\
\cline { 2 - 7 } & $\begin{array}{c}\text { Intelectualmente } \\
\text { Deficiente }\end{array}$ & $20.21 \%$ & 19 & 3 & $3.19 \%$ & Muy baja & \\
\hline
\end{tabular}

Nota: $* \mathrm{~N}=$ número de participantes. $* * \sum=$ sumatoria de porcentajes.

Se puede observar que las puntaciones del WISC-III v.ch., en comparación con el TONI-2, arrojaron porcentajes menores en las clasificaciones: promedio, media alta, superior y muy superior. Sin embargo, los porcentajes del WISC-III v.ch. fueron mayores en las clasificaciones media baja, limítrofe e intelectualmente deficiente. En relación a lo anterior, el WISC-III v.ch. clasificó a un 32\% de la muestra por sobre el rango medio bajo y a un $68 \%$ por debajo del promedio. No obstante, el TONI-2 clasificó a un $67 \%$ de la muestra sobre el rango medio bajo y a un 33\% bajo el promedio.

En la siguiente tabla se pueden apreciar los promedios de cada escala e índice del WISC-III v.ch. y el promedio del TONI-2. 
Tabla 4. Puntajes promedio de WISC-III v.ch. y TONI-2

\begin{tabular}{|l|c|c|c|}
\hline \multicolumn{1}{|c|}{ WISC-III v.ch. } & $\mathrm{N}$ & Media & Desviación típica \\
\hline CI Total & 94 & 82.66 & 19.03 \\
CI Verbal & 94 & 75.52 & 15.73 \\
CI Ejecución & 94 & 94.49 & 22.56 \\
Comprensión Verbal & 94 & 71.82 & 13.77 \\
Organización Perceptual & 94 & 82.74 & 16,12 \\
Velocidad Procesamiento & 94 & 86.36 & 13.94 \\
Ausencia Distractibilidad & 94 & 76.39 & 15.65 \\
\hline \multicolumn{1}{|c|}{ TONI-2 } & $\mathrm{N}$ & Media & Desviación típica \\
\hline CI Total & 94 & 97.38 & 15.16 \\
\hline
\end{tabular}

En la Tabla 5 se pueden ver los resultados del contraste de diferencias de medias para ambos instrumentos.

Tabla 5. Diferencias de medias muestra versus norma

\begin{tabular}{|l|c|c|c|c|}
\hline \multicolumn{1}{|c|}{ WISC-III v.ch. } & $t$ & $\mathrm{gl}$ & $p$ & Diferencia medias \\
\hline CI Total & -8.83 & 93 & .000 & -17.34 \\
CI Verbal & -15.09 & 93 & .000 & -24.48 \\
CI Ejecución & -2.37 & 93 & .020 & -5.51 \\
Comprensión Verbal & -19.85 & 93 & .000 & -28.18 \\
Organización Perceptual & -10.38 & 93 & .000 & -17.26 \\
Velocidad Procesamiento & -9.49 & 93 & .000 & -13.64 \\
Ausencia Distractibilidad & -14.66 & 93 & .000 & -23.61 \\
\hline \multicolumn{1}{|c|}{ TONI-2 } & $t$ & $\mathrm{gl}$ & $p$ & Diferencia medias \\
\hline CI Total & -1.67 & 93 & .098 & -2.62 \\
\hline
\end{tabular}

En la Tabla 5 se evidencia que la media de la Escala Total del WISC-III v.ch. es de 82,66 puntos, en la Escala Verbal la media es de 75,52 puntos, en la Escala de Ejecución la media es de 94,49 puntos. Con respecto a los índices del WISC-III v.ch., la media para el índice de Comprensión Verbal es de 71,82 puntos, en el índice de Organización Perceptual la media es de 82,74 puntos, en el índice de Velocidad de Procesamiento la media es de 86,36 puntos y, por último, el índice de Ausencia de Distractibilidad es de 76,39 puntos. Con esta información, más la información proporcionada por la Tabla 4, se puede observar que el rendimiento de los niños de zona rural es significativamente más bajo en comparación con la norma poblacional establecida por el WISC-III v.ch., ya que se encontraron diferencias 
Estudios Pedagógicos XLII, N 3: 413-427, 2016

APROXIMACIÓN COMPARATIVA INICIAL EN RESULTADOS DEL WISC-III V.CH. ENTRE UNA MUESTRA DE JÓVENES ESCOLARIZADOS PERTENECIENTES A ZONAS RURALES DE LA XV REGIÓN DE ARICA Y PARINACOTA...

estadísticamente significativas en las Escala Completa, Verbal, Ejecución e Índices de Comprensión Verbal, Organización Perceptual, Velocidad de Procesamiento y Ausencia de Distractibilidad.

Por otra parte, al comparar la media obtenida por la muestra en el TONI-2 (97.38) con su respectiva norma, no se observan diferencias estadísticamente significativas.

\section{DISCUSIÓN}

El objetivo general de esta investigación consistió en comparar resultados del WISCIII v.ch entre una muestra rural de la XV Región de Arica y Parinacota y la norma nacional. Además, se decidió incluir una medición adicional de coeficiente intelectual, el test de inteligencia no verbal TONI-2, dado que no se encuentra afecto a sesgo cultural.

De acuerdo con los hallazgos de esta investigación quedaron en evidencia diferencias estadísticamente significativas en el rendimiento entre los jóvenes rurales muestreados y la norma, en cada una de las escalas e índices de la versión chilena del WISC-III. Por otro lado, con respecto al TONI-2, no se hallaron diferencias estadísticamente significativas en el coeficiente intelectual.

Con todo esto, los resultados obtenidos a través de la versión chilena del WISC-III demostraron que más de la mitad de los jóvenes que viven zonas rurales de la XV Región de Arica y Parinacota presentaron un CI por debajo del promedio, además, la mayoría de estos puntuaron en las categorías limítrofe e intelectualmente deficiente. Sin embargo, cuando el CI fue medido a través del TONI-2, los resultados son completamente diferentes, más de la mitad de los participantes obtuvo un CI dentro y sobre la media.

Según los resultados obtenidos en la evaluación realizada con el WISC-III v.ch., los jóvenes muestreados, pertenecientes al área rural, presentan promedios notablemente más bajos que los jóvenes predominantemente urbanos y con más capacidad competitiva, especialmente en la escala verbal y el índice de comprensión verbal, los cuales se encuentran dentro de la categoría limítrofe. Se considera que estas escalas del WISC-III v.ch. dan cuenta general de la habilidad de los niños para comprender el lenguaje, procesar la información presentada por vía verbal y organizar una respuesta. Cabe mencionar, que estas escalas están estrechamente relacionadas con las posibilidades educativas, así que su interpretación debe hacerse teniendo en cuenta el conocimiento sobre el tipo de educación que recibe el niño.

Estos resultados pueden deberse a las características de la muestra en términos socio culturales, medioambientales y educacionales, donde es importante resaltar que las posibilidades educativas de estos jóvenes pueden verse disminuidas en comparación con la población predominantemente urbana, debido a variables ambientales y eco-culturales como la condición de ruralidad, distancia y la ubicación geográfica en la que se encuentran, más aun cuando gran parte de la muestra vive en estancias aisladas, con difícil acceso a educación preescolar, la que se encuentra únicamente en la capital de la comuna y solo tienen acceso a una escuela, las cuales en su mayoría son unidocentes y de bajo número de alumnos (entre tres y diez), implicando que estudiantes de distintos niveles compartan, al mismo tiempo, la misma aula y profesor. En este sentido, se ha encontrado que algunas de estas variables ambientales están consistentemente relacionadas con la inteligencia y el funcionamiento cognitivo (Georgas et al., 2003). Como ejemplo, algunos de los profesores 
a cargo de estas escuelas manifestaron que existe una baja escolaridad de los padres, además su principal actividad es el pastoreo o la agricultura, dependiendo de la localidad, esto implica que, como se pudo observar en terreno, varios de estos niños pasan gran parte de los días solos y en precarias condiciones.

Es importante señalar que en la estandarización chilena del WISC-III se afirma que tanto la magnitud de la muestra como el hecho que se base en un muestreo estratificado (p. ej.: por regiones del país) permiten confiar en que las normas son representativas de las diferentes especificidades subculturales que puedan existir en el país (Ramírez y Rosas, 2007), aunque en el muestreo no se incluyó ninguna localidad rural.

Debido a lo planteado anteriormente, a los hallazgos presentados en esta investigación y a las características de la muestra, creemos que es pertinente abrir una discusión al respecto de estas afirmaciones. Los resultados obtenidos pueden deberse a dos fuentes. La primera apunta a que el instrumento WISC-III v.ch. en su parte verbal es inadecuado para medir la capacidad intelectual de los niños, en ese caso la solución es simple, ya que implicaría cambiar de instrumento. La segunda fuente de explicación es más compleja, y es que estos resultados se deban a que un grupo importante de niños está en una situación abiertamente deficitaria, que se traduciría en un rendimiento intelectual verbal inferior a la media, situación que se estaría produciendo por la variables que hemos mencionado anteriormente, tales como bajo nivel de escolaridad de los padres, baja educación preescolar y deficientes condiciones en la educación básica. La presente investigación no puede dar una respuesta certera a este dilema, ya que se limita a constatar un hecho, los niños de sectores rurales tienen un bajo CI verbal, medido con el WISC-III v.ch. No obstante, en el supuesto de que estos resultados se deban a la segunda fuente mencionada, tendrían importantes implicancias prácticas en el sentido que los niños (as) y jóvenes de áreas rurales se encontrarían en una situación de desventaja respecto de las habilidades cognitivas evaluadas por el WISC III v.ch., máxime las que se asocian al componente verbal, en comparación con los niños y jóvenes urbanos. De esto se desprende que esta sub-población no estaría recibiendo la estimulación necesaria para enfrentar las metas, a corto y largo plazo, que el sistema educacional exige. En esta línea, podemos señalar se deberían realizar intervenciones destinadas a la estimulación intelectual en edades tempranas, momento en que es posible conseguir mejores resultados, debido a que la capacidad intelectual se encuentra en pleno desarrollo. Asimismo, se recomienda que las intervenciones incluyan a los padres, ya que al tener una baja escolaridad no brindarían la estimulación necesaria a los niños (as) y jóvenes. Estas intervenciones debieran estar enmarcadas dentro de una rigurosa valoración de programas, con mediciones antesdespués y su respectivo seguimiento, con el objetivo de poder contar con resultados sobre su eficacia y hacer ajustes de ser necesario.

En relación con lo anteriormente señalado, como psicólogos tenemos una tarea ineludible en esta situación. Las universidades que imparten la carrera de psicología a lo largo de todo el país debieran incluir dentro de alguna asignatura talleres prácticos, o bien prácticas intermedias, en que los alumnos tuvieran que aplicar un programa de estimulación intelectual. Con esta aplicación masiva, y su correspondiente valoración, podríamos contribuir, en parte, a disminuir la brecha entre los chicos y chicas rurales y urbanos, otorgándoles mayores probabilidades de éxito en el futuro. Cabe hacer notar que los alumnos de psicología también se verían beneficiados al desarrollar o fortalecer competencias profesionales que son sustantivas a nuestro quehacer. 
Estudios Pedagógicos XLII, N 3: 413-427, 2016

APROXIMACIÓN COMPARATIVA INICIAL EN RESULTADOS DEL WISC-III V.CH. ENTRE UNA MUESTRA DE JÓVENES ESCOLARIZADOS PERTENECIENTES A ZONAS RURALES DE LA XV REGIÓN DE ARICA Y PARINACOTA...

En tanto, los profesionales que utilizan este instrumento deberían ser conscientes de las influencias medioambientales en los puntajes de los test, e incorporarlas dentro de sus interpretaciones según corresponda para cada evaluación. Por consiguiente, y mientras no se disponga de más réplicas sobre este tema, creemos necesario dar énfasis a la integración de escalas de índole no verbal para dar respuesta a la evaluación de individuos con características similares a la muestra de esta investigación.

Dentro de las limitaciones del presente estudio se puede mencionar el difícil acceso a la muestra, debido a la distancia geográfica entre las distintas localidades, motivo que llevó a disminuir el tamaño muestral que inicialmente se consideraba.

Invitamos a la comunidad científica de las diferentes regiones del país a continuar esta línea de trabajo y contrastar los hallazgos que presentamos. Destacamos el carácter provisional de los resultados presentados y la necesidad de mayor investigación al respecto.

\section{REFERENCIAS BIBLIOGRÁFICAS}

Adriazola, A., Ascencio, S., Bernales, M., Carrasco, C., Keller, A., Polanco, F., Salas, C., \& Santelices, R. (1976). Estandarización de la Escala Revisada de Wechsler para la medición de la inteligencia en los niños (WISC-R) (Memoria para optar al título de Psicólogo). Pontificia Universidad Católica de Chile, Santiago, Chile.

Anastasi, A., \& Urbina, S. (1997). Psychological testing (7th ed.). Upper Saddle River, NJ: PrenticeHall.

Ardila, R. (2011). Inteligencia. ¿Qué sabemos y qué nos falta por investigar? Revista Académica Colombiana de Ciencias, 35(134), 97-103.

Berry, J.W., Poortinga, Y.H., Segall, M.H., \& Dasen, P.R. (2002). Cross-cultural psychology: Research and applications. Cambridge, UK: Cambridge University Press.

Bracken, BA., \& McCallum, R.S. (1997). The Universal Test of Nonverbal Intelligence. Chicago, IL: Riverside Publishing.

Brown, L., Sherbenou, R.J., \& Johnsen, S.K. (1995). Test de inteligencia no Verbal Toni-2. Madrid: TEA.

Calderón, M., Castillo, M., Mandujano, L., Pérez, C., \& Purcell, C. (1980). Estandarización de la Escala Revisada de Inteligencia de Wechsler para niños chilenos del Área Metropolitana (WISC-R). Tercera etapa (Memoria para optar al título de Psicólogo). Pontificia Universidad Católica de Chile, Santiago, Chile.

Cañas, M., Colzani, M., Domínguez, A., Domínguez, M., Jorquera, K., Orpinas, P., \& Valdivieso, P. (1978). Adaptación, análisis estadístico y construcción de normas de la Escala Revisada de Inteligencia de Wechsler para niños entre 6 años 0 días y 9 años 11 meses 30 días (WISC-R) (Memoria para optar al título de psicólogo). Pontificia Universidad Católica de Chile, Santiago, Chile.

Cayssials, A. (1998). La Escala de Inteligencia WISC-III en la evaluación psicológica infantojuvenil. Buenos Aires: Paidós.

Cronbach, L.J. (1990). Essentials of Psychological Testing. New York. Harper \& Row Publishers.

Frank de Verthelyi, R. (2000). Las inteligencias y la evaluación: Interrogantes y tendencias actuales. Psicodebate. Psicología, Cultura y Sociedad, 1(1), 107-116. Recuperado de https://dspace. palermo.edu:8443/dspace/handle/10226/375

Georgas, J., Waiss, L., van de Vijver, F., \& Saklofske, D. (2003). Culture and Children's Intelligence. San Diego: Academic Press.

Grubb, H. (1983). The Cultural Distance Approach: A Model for Analyzing Black-White Performance Differences on Measures of IQ. Recuperado de http://files.eric.ed.gov/fulltext/ED240192.pdf 
Hopper, B. C. (2000). Examining the concurrent and predictive validity of nonverbal tests of intelligence. (Order No. 3044309, George Fox University). ProQuest Dissertations and Theses. Jensen, A.R. (1980). Bias in mental testing. New York: Free Press.

Kezer, F., \& Arik, R. (2012). An examination and comparison of the revisions of the Wechsler Intelligence Scale for Children. Procedia-Social and Behavioral Sciences, 46, 2104-2110. doi:10.1016/j.sbspro.2012.05.436

Kowall, M., Watson G., \& Madak P. (1990). Concurrent Validity of the Test of Nonverbal Intelligence with Refered Suburban and Canadian Native Children. Journal of Clinical Psychology, 46(5), 632-636. doi:10.1002/1097-4679(199009)46:5<632::AID-JCLP2270460515>3.0.CO;2-8

Lopez, E. C., Lamar, D., \& Scully-Demartini, D. (1997). The cognitive assessment of limited-englishproficient children: Current problems and practical recommendations. Cultural Diversity and Mental Health, 3(2), 117-130.

Martínez, S. (2009). WISC III: diferencias entre las tablas estadounidenses y las montevideanas. Posibles implicancias diagnósticas. Artículo presentado en el 1er. Congreso de Diagnóstico e Intervención Psicológica IV Jornadas del Área de Diagnóstico e Intervención Psicológica. Caleidoscopio de Prácticas. Recuperado de http://www.psico.edu.uy/sites/default/files/cursos/ exploracionaspectos_trabajo-congreso.pdf

Matarazzo, J. (1972). Wechsler's measurement and appraisal of adult intelligence ( $5^{\mathrm{a}}$ ed.). Baltimore: Williams \& Wilkins.

Miller, J.G. (1997). A cultural psychology perspective on intelligence. In R.J. Sternberg \& E. Rigorenko (Eds.), Intelligence, heredity and environment (pp. 269-302). Boston: Cambridge University Press.

Ramírez, V., \& Rosas, R. (2007). Estandarización del WISC-III en Chile, Estructura Factorial y Consistencia Interna de las Escalas. PSYKHE, 16(1), 91-109.

Roid, G.H., \& Miller, L.J. (1997). Leiter International Performance Scale-Revised. Wood Dale, IL: Stoelting Co.

Sattler, J.M. (1992). Assessment of children (revised and updated, 3rd ed.). San Diego, CA: Author.

Savaşır, I., \& Şahin, N. (1995). Wechsler Çocuklar İçin Zeka Ölçeği (WISC-R) [Escala de Inteligencia Wechsler para Niños]. Türk: Psikologlar Derneği.-

Sternberg, R.J. (1992). CAT: A program of comprehensive abilities testing. In Guifford, B., \& O'Connor, M.C. (Eds.). Changing Assessments. Alternative views of aptitude, achievement and instruction (pp. 213-274). Boston: Kluwer Academic Publishers.

Toker, F. (1968). Zeka Kuramlari [Teorías de Inteligencia]. Ankara: Milli Egitim Bakanligi Talim ve Terbiye Dairesi Arastirma ve Degerlendirme Burosu.

Van Hemert, D.A. (2003). Cross-Cultural Meta-Analyses. Online Readings in Psychology and Culture, 2(2). Recuperado de: http://scholarworks.gvsu.edu/orpc/vol2/iss2/5/

Walsh W.B., \& Betz, N.E. (1995). Test and Assessment. New Jersey. Prentice-Hall, Englewood Cliffs. Wechsler, D. (1991). Wechsler Intelligence Scale for Children (3rd ed). San Antonio, TX: The Psychological Corporation.

Wechsler, D. (1997). WAIS-III, WMS-III, technical manual. San Antonio, TX: The Psychological Corporation 
